ISSN 1112-9867

http://www.jfas.info

\title{
NUMERICAL SIMULATION OF BRAIN TUMOR GROWTH MODEL USING TWO-STAGE GAUSS-SEIDEL METHOD
}

\author{
A. Hussain*, M. S. Muthuvalu and I. Faye \\ Department of Fundamental and Applied Sciences, UniversitiTeknologi PETRONAS, 32610 \\ Bandar Seri Iskandar, Perak, Malaysia
}

Published online: 10 November 2017

\begin{abstract}
In the recent years, the study of glioma growth continuous to be an active field of research. Mathematical models that describe the proliferation and diffusion properties of the glioma growth have been developed by many researchers. In this work, the performance analysis of two-stage Gauss-Seidel (TSGS) method to solve the glioma growth model is investigated. The governing model is discretized by using Backward Time Central Space (BTCS) scheme. The proposed method is developed by using MATLAB software and some numerical simulations have been conducted. The findings show that the TSGS method is better than the standard Gauss-Seidel (GS) method in terms of number of iterations and computational time.
\end{abstract}

Keywords: backward time central space scheme; Gauss-Seidel method; two-stage Gauss-Seidel method.

Author Correspondence, e-mail: abidahussain490@yahoo.com

doi: http://dx.doi.org/10.4314/jfas.v9i6s.18 


\section{INTRODUCTION}

Glioma is the most invasive brain tumor, arising from the abnormal growth of glial cells in thebrain [1]. To study the dynamics of brain glioma, researchers develop the mathematical model.Most of the mathematical model of brain tumor growth is in the form of partial differential equation (PDE) [2]. More specifically, the second order reaction-diffusion equation is used widely to model the glioma growth [3]. Generally, finite difference (FD) method is applied widely to solve the PDE [4-7]. An implementation of FD method will leads to an algebraic system with the sparse coefficient matrix.

Numerical methods to solve the algebraic system are categorized into two main classes,i.e. directand iterative methods [8]. However, for large sparse systems, the limitations of direct methods are enormous computational time and slow convergence rate. Consequently, the iterative methods are preferred than direct methods to solve the large sparse algebraic systems [7]. Among the existing iterative methods, two-stage methods are widely used and efficient compared to one-stage methods. A two-stage iterative method often called inner-outer iteration method and first proposed by [9]. Many researches have been conducted to investigate the effectiveness of two-stage methods, refer [10-17].

In this work, the effectiveness of two-stage Gauss-Seidel (TSGS) method for solving large sparse linear algebraic system generated from the discretization of glioma growth model will be investigated. The considered glioma growth model will be discretized by using FD method. The performance of the tested TSGS method will be compared with standard Gauss-Seidel (GS) method.

The outline of the paper is as follows: the mathematical model of glioma growth is explained in Section 2. Section 3 describes the numerical methods used to solve the governing model. Numerical results are included and discussed in Section 4. Finally, the concluding remarks are given in Section 5.

\section{MATHEMATICAL MODEL OF BRAIN TUMOR GROWTH}

The reaction-diffusion model introduce by [2] had a significant impact on glioma growth modeling. The basic model considers the evolution of glioma tumor cells population to be mainly governed by proliferation and diffusion. Initially, the growth of an infiltrating glioma 
as a mass conservation law was provided by [2]. Mathematically, the model can be represented as

$$
\frac{\partial c}{\partial t}=\nabla \cdot J+f(c), \quad x \in \Omega
$$

where $c$ designates the tumor cell density at location $x$ and time $t, f(c)$ as the function of cell density, $\nabla$ defines the spatial gradient operator and $\Omega$ is the brain domain. Under the assumption of classical Flick's law, the following relation satisfies

$$
J=D \nabla c
$$

where $D$ is the diffusion coefficient.

Three different cancerous growth such as exponential, logistic and Gompertz are used for $f(c)$ [2]. Brain tumor growth is commonly assumed to be exponential, with constant growth rate. If the cell density reached an exponentially high level, then the logistic or Gompertzian model was more reasonable. However, in the case of glioma growth, the exponential growth is frequently used as the tumor become fatal before the cell density reached the significant high intensity. For this reason, the exponential growth is still sound acceptable with the time frame considered. Based on the Equation (2) and $f(c)=\rho c$, the Equation (1) can be rewritten as

$$
\frac{\partial c}{\partial t}=D \nabla^{2} c+\rho c
$$

with initial condition

$$
c(x, 0)=f(c)
$$

and Neumann boundary condition

$$
n \cdot D \nabla c(x, t)=0
$$

where $\rho$ is the proliferation rate.

\section{NUMERICAL METHODS}

In this section, the implementation of numerical methods used to solve the glioma growth model as given in Equation (3) is explained in details. The Backward Time Central Space 
(BTCS) scheme is applied to discretize the model (3) and then the resulting linear algebraic system will be solved by using GS and TSGS methods.

\subsection{Backward Time Central Space (BTCS) Scheme}

Let the solution domain be divided uniformly in both $x$ and $t$. Thus, the discrete set of points of $x$ and $t$ respectively be given by $x_{i}=i \Delta x(i=0,1,2, \ldots, n-2, n-1, n)$ and $t_{s}=s \Delta t$ $(s=0,1,2, \ldots, m-2, m-1, m)$. For simplicity, the notation $c_{i, s} \equiv c\left(x_{i}, t_{s}\right)$ will be used subsequently.

For the implementation of BTCS scheme, the following formula are considered

$$
\frac{\partial c}{\partial t}=\frac{c_{i, s+1}-c_{i, s}}{\Delta t}+O(\Delta t)(4)
$$

and

$$
\frac{\partial^{2} c}{\partial x^{2}}=\frac{c_{i-1, s+1}-2 c_{i, s+1}+c_{i+1, s+1}}{(\Delta x)^{2}}+O\left(\Delta x^{2}\right)
$$

The implementation of formula given in Equations (4) and (5) into Equation (3) will reduces the model to

$$
c_{i, s}=-q c_{i-1, s+1}+p c_{i, s+1}-q c_{i+1, s+1}(6)
$$

where $p=\frac{1+2 \beta}{1+\alpha} \quad$ and $\quad q=\frac{\beta}{1+\alpha}$ with $\quad \alpha=\frac{\rho L^{2}}{D} \Delta t \quad$ and $\quad \beta=\frac{\Delta t}{(\Delta x)^{2}}$.Moreover, the approximation equation (6) can be rewritten in matrix form as

$$
A c=b(7)
$$

where

$$
A=\left[\begin{array}{ccccccc}
p & -q & & & & & \\
-q & p & -q & & & 0 & \\
& -q & p & -q & & & \\
& & \ddots & \ddots & \ddots & & \\
& & & -q & p & -q & \\
& 0 & & & -q & p & -q \\
& & & & & -q & p
\end{array}\right], c=\left[\begin{array}{c}
c_{1, s+1} \\
c_{2, s+1} \\
c_{3, s+1} \\
\vdots \\
c_{n-3, s+1} \\
c_{n-2, s+1} \\
c_{n-1, s+1}
\end{array}\right] \text { and } b=\left[\begin{array}{c}
c_{1, s}+q c_{0, s+1} \\
c_{2, s} \\
c_{3, s} \\
\vdots \\
c_{n-3, s} \\
c_{n-2, s} \\
c_{n-1, s}+q c_{n, s+1}
\end{array}\right] .
$$

\subsection{GS and TSGS methods}

In this section, the formulation of GS and TSGS methods will be explained. Now, consider 
the splitting [18]

$$
A=P-Q, \quad P=M-N
$$

where $M$ is the diagonal matrix, $N$ is the strictly lower triangular matrix and $Q$ is the strictly upper triangular matrix. Theiterative form of GS and TSGS methods are

$$
c^{(k+1)}=T c^{(k)}+d, T=P^{-1} Q, d=P^{-1} b
$$

and

$$
c^{(k+1)}=\left(M^{-1} N\right)^{j} c^{(k)}+\sum_{i=0}^{j-1}\left(M^{-1} N\right)^{i} M^{-1}\left(Q c^{(k)}+b\right), k=0,1,2, \cdots,
$$

respectivelywhere $j$ is the inner iteration number, and $i$ is the outer iteration. In the case of the stationary two-stage method, inner iteration number is fixed and $j \geq 1$. Based on the theorem, the both methods will converge if the spectral radius is less than one. The algorithm of GS and TSGS are described in algorithms 1 and 2 respectively.

\section{Algorithm 1. One-Stage Gauss-Seidel Method}

Step 1. Initialize all the parameter.

Step 2. while $k=1,2,3, \ldots$ until convergence

$$
\begin{aligned}
& \text { for } s=1,2,3, \ldots, m-3, m-2, m-1, m \\
& \quad \text { for } i=1,2,3, \ldots, n-3, n-2, n-1 \\
& c_{i, s}^{(k+1)}=P^{-1} Q c_{i, s}^{(k)}+P^{-1} b
\end{aligned}
$$

Step 3. Convergence test. If the convergence criterion i.e. $\left\|c^{(k+1)}-c^{(k)}\right\|<\varepsilon$ (where $\varepsilon$ is convergence criterion) is satisfied, go to step 4. Otherwise, go to step 2.

Step 4. Stop.

\section{Algorithm 2. Two-Stage Gauss-Seidel Method}

Step Initialize all the parameter.

1.

Step while $k=1,2,3, \ldots$ until convergence

2. 


$$
\begin{aligned}
\text { for } s= & 1,2,3, \ldots, m-3, m-2, m-1, m \\
& \text { for } i=1,2,3, \ldots, n-3, n-2, n-1 \\
y_{0, s}^{(k)}= & c_{i-1, s}^{(k)} \\
& \text { for } j=1 \text { to } l \\
y_{j, s}^{(k+1)}= & M^{-1} N y_{j-1, s}^{(k)}+M^{-1} Q c_{i-1, s}^{(k)}+M^{-1} b \\
c_{i, s}^{(k+1)}= & y_{j, s}^{(k+1)}
\end{aligned}
$$

Step Convergence test. If the convergence criterion i.e. $\left\|c^{(k+1)}-c^{(k)}\right\|<\varepsilon$ (where $\varepsilon$ is 3. convergence criterion) is satisfied, go to step 4. Otherwise, go to step 2.

Step Stop.

4.

\section{RESULTS AND DISCUSSION}

To perform the numerical experiments, the initial and boundary conditions, i.e., $f(c)=4000$ cells and no flux boundary respectively are considered. Meanwhile, the value of diffusion coefficient, $D$ is $0.0013 \mathrm{~mm}^{2} \mathrm{day}^{-1}$ and the growth rate, $\rho$ is $0.012 \mathrm{day}^{-1}$ are considered[6]. The parameter such as number of iterations $(k)$, computational time in seconds (Time) and maximum cell density $\left(c_{\max }\right)$ are measured for the performance analysis of the GS and TSGS methods. The value of initial iteration is set as zero for both methods. All the simulations are developed by using MATLAB and performed on a personal computer with Intel(R) Core(TM) i7- 2600 CPU@ (3.40 GHz, 3.40 GHz) and 8.00 GB RAM. The numerical results are considered until 15 days of growth with convergence test for tolerance error $\varepsilon=10^{-6}$. The numerical results of GS and TSGS methods are presented in Tables 1 to 3. For TSGS method the value of inner iteration is fixed, i.e. $l=10$. The numerical simulations are carried out for four different mesh sizes, i.e. $30,60,120$ and 240. 
Table 1.Numerical results for $s=5$ days

\begin{tabular}{ccccc}
\hline $\boldsymbol{n}$ & Methods & $\boldsymbol{k}$ & Time & $\boldsymbol{c}_{\max }$ \\
\hline 30 & GS & 2712 & 112.25 & 4364 \\
& TSGS & 1623 & 16.28 & 4355 \\
60 & GS & 10045 & 126.16 & 4413 \\
& TSGS & 5640 & 25.08 & 4412 \\
120 & GS & 36714 & 203.32 & 4439 \\
& TSGS & 19047 & 53.38 & 4424 \\
\hline \multirow{2}{*}{240} & GS & 132766 & 1975.50 & 4443 \\
& TSGS & 62038 & 194.33 & 4420 \\
\hline
\end{tabular}

Table 2.Numerical results for $s=10$ days

\begin{tabular}{ccccc}
\hline $\boldsymbol{n}$ & Methods & $\boldsymbol{k}$ & Time & $\boldsymbol{c}_{\max }$ \\
\hline 30 & GS & 1510 & 109.14 & 4634 \\
& TSGS & 925 & 20.07 & 4584 \\
60 & GS & 5571 & 163.77 & 4672 \\
& TSGS & 3230 & 29.37 & 4617 \\
120 & GS & 20409 & 355.02 & 4682 \\
& TSGS & 11039 & 72.97 & 4624 \\
\hline
\end{tabular}


Table 3.Numerical results for $s=15$ days

\begin{tabular}{ccccc}
\hline $\boldsymbol{n}$ & Methods & $\boldsymbol{k}$ & Time & $\boldsymbol{c}_{\max }$ \\
\hline 30 & GS & 1055 & 120.89 & 4721 \\
& TSGS & 655 & 22.33 & 4630 \\
60 & GS & 3888 & 179.84 & 4750 \\
& TSGS & 2292 & 33.08 & 4653 \\
120 & GS & 14262 & 450.72 & 4758 \\
& TSGS & 7884 & 87.64 & 4658 \\
240 & GS & 51928 & 1433.80 & 4757 \\
& TSGS & 26422 & 347.27 & 4657 \\
\hline
\end{tabular}

From the numerical results obtained, the number of iterations for TSGS method reduced approximately, $40.15-53.27 \%$ (for 5 days), $38.74-50.57 \%$ (for 10 days) and $37.91-49.11 \%$ (for 15 days) respectively as compared to GS method. The execution of TSGS method is faster approximately by $73.74-90.12 \%$ (for 5 days), $65.83-82.06 \%$ (for 10 days) and $75.77-81.60 \%$ (for 15 days) as compared to GS method.

\section{CONCLUSION}

In this work, numerical solutions of one-dimensional glioma growth model is considered.From the Tables 1 to 3, it can be clearly seen that TSGS method required less number of iterations and computational time as compared to GS iterative method. For the future works, the numerical solutions obtained can be validated with the real clinical data. Also, higher order model can be considered to predict glioma growth.

\section{ACKNOWLEDGEMENTS}

The authors would like to thank UniversitiTeknologi PETRONAS Graduate Assistantship Scheme and Center of Intelligent Signal and Imaging Research (CISIR) for support in conducting this research. 


\section{REFERENCES}

[1] Swanson KR, Alvord EC, Murray JD. Mathematical and computer modelling.2003, 37(11):1177-1190

[2] Murray J.D. Mathematical biology II:Spatial models and biomedical applications \{Interdisciplinary Applied Mathematics V. 18\}.New York: SpringerVerlag, 2001

[3] Roniotis A, Marias K, Sakkalis V, Stamatakos G, Zervakis M. Comparing finite elements and finite differences for developing diffusive models of glioma growth. InAnnual International Conference of the IEEE Engineering in Medicine and Biology Society,2010, pp. $6797-6800$

[4] Pheng H S, Alias N, Said N M. High performance simulation for brain tumours growth using parabolic equation on heterogeneous parallel computer system. Asia-Pacific Journal of Information Technology and Multimedia, 2007, 4(1): 39-52

[5] Özuğurlu E. A note on the numerical approach for the reaction-diffusion problem to model the density of the tumor growth dynamics. Computers and Mathematics with Applications, 2015, 69(12):1504-1517

[6] Tracqui P, Cruywagen G C, Woodward D E, Bartoo G T, Murray J D, Alvord E C. A mathematical model of glioma growth: The effect of chemotherapy on spatio-temporal growth. Cell Proliferation, 1995, 28(1):17-31

[7] Hussain A, Faye I, Muthuvalu M S. Performance analysis of successive over relaxation method for solving glioma growth model. AIP Conference Proceedings, 2016, 1787(1):1-6

[8] Saad Y. Iterative methods for sparse linear systems. Pennsylvania: Society for Industrial and Applied Mathematics, 2003

[9] Nichols NK. On the convergence of two-stage iterative processes for solving linear equations. SIAM Journal on Numerical Analysis, 1973, 10(3):460-469

[10] Szyld DB, Jones MT. Two-stage and multisplitting methods for the parallel solution of linear systems. SIAM Journal on Matrix Analysis and Applications, 1992, 13(2):671-679

[11] Frommer A, Szyld DB. H-splittings and two-stage iterative methods. NumerischeMathematik, 1992, 63(1):345-356

[12] Frommer A, Szyld D B. Asynchronous two-stage iterative methods. NumerischeMathematik, 1994, 69(2):141-153 
[13] Galligani E, Ruggiero V. The two-stage arithmetic mean method. Applied Mathematics and Computation, 1997, 85(2):245-264

[14] Bai ZZ. The convergence of the two-stage iterative method for Hermitian positive definite linear systems. Applied Mathematics Letters, 1998, 11(2):1-5

[15] Cao ZH. Convergence of nested iterative methods for symmetric P-regular splittings. SIAM Journal on Matrix Analysis and Applications, 2000, 22(1):20-32

[16] Bai ZZ, Wang CL. On the convergence of nonstationarymultisplitting two-stage iteration methods for hermitian positive definite linear systems. Journal of Computational and Applied Mathematics, 2002, 138(2):287-296

[17] Yun JH, Kim SW. Convergence of two-stage iterative methods using incomplete factorization. Journal of Computational and Applied Mathematics, 2004, 166(2):565-580

[18] Berman A, Plemmons RJ. Nonnegative matrices in the mathematical sciences. Pennsylvania:Society for Industrial and Applied Mathematics; 1994

\section{How to cite this article:}

Hussain A, Muthuvalu M S, Faye I. Numerical Simulation Of Brain Tumor Growth Model Using Two-Stage Gauss-Seidel Method. J. Fundam. Appl. Sci., 2017, 9(6S), 227-236 